\title{
IMPROVING THE ACCURACY OF SURGE MODELS USING SUBGRID CORRECTIONS
}

\author{
Andrew Kennedy, University of Notre Dame, Andrew.kennedy@nd.edu \\ Damrongsak Wirasaet, University of Notre Dame, dwirasae@nd.edu \\ Diogo Bolster, University of Notre Dame, dbolster@nd.edu \\ J. Casey Dietrich, North Carolina State University, jcdietrich@ncsu.edu
}

\section{INTRODUCTION}

Modern storm surge models to predict hurricane water levels have gone in two opposite directions: (1) Low resolution, fast, models that may be run thousands of times as a storm approaches land; and (2) High resolution, more accurate, models that are largely used for planning and hindcasts, and are too slow for real-time ensemble forecasts. Differences in predictions between the two types of models are particularly large over flooded ground, which is most important for human activities.

\section{SUBGRID CORRECTIONS}

A methodology to improve performance at the water's edge was suggested by Casulli (2009), who improved wetting and drying by using high resolution, subgrid, topographies to improve representations of cell volume and boundary fluxes as a function of surface elevation. These subgrid integrals may be computed prior to running the surge model, and then used to introduce corrections to surge computations at low resolution. The corrections for volume were found to strongly improve wetting and drying in coastal regions when compared to standard solutions.

This paper will derive and test improvements to Casulli's subgrid techniques which include representations of bottom friction, wind stress, flow connectivity, and convective stresses, and will present real-world examples with comparisons in accuracy and efficiency.

\section{MATHEMATICAL APPROACH}

For a specified bathymetric depth function, $b(x, y)$, the porosity at a specific elevation, $Z$, is given as

$$
p(x, y, z)= \begin{cases}0, & b(x, y)+z \leq 0 \\ 1, & b(x, y)+z>0\end{cases}
$$

An overall cell porosity at a given elevation is then the integral of the porosity function over that cell. In partiallyfilled cells, this greatly improves representations of wetting and drying through a slightly-modified massconservation equation,

$$
\frac{\partial H_{i j}}{\partial t}+\frac{1}{p_{i j}} \nabla \cdot\left[H_{i j} \mathbf{U}_{i j}\right]=0 .
$$

The $p_{i j}$ function is the subgrid-based representation of cell porosity, which varies strongly with elevation and allows higher resolution properties to be introduced into the lower resolution grid. Similar, but much more complex, techniques may be introduced to improve bottom friction, continuity properties, and convective momentum transfer using subgrid properties integrated to larger scale.

\section{EXAMPLE}

Figure 1 shows a preliminary example for tidal wetting and drying of Buttermilk Bay, MA. High resolution time series are compared to low resolution solutions for both subgrid and standard solutions. In the main bay at Station 13, low-resolution subgrid time series are much more accurate than their standard counterparts, although each shows some error. At Station 22, which is in a secondary bay connected to the main bay by a narrow channel, subgrid solutions show some error; however, standard solutions will not even fill the bay in this case because they cannot represent the narrow connection between bays. At the conference we will show additional examples and development over the past year.

\section{CONCLUSIONS}

Subgrid corrections to coarse resolution surge models can greatly increase accuracy when compared to standard solutions. These will not only prove useful in improving operational storm ensemble forecasts, but will also enable new types of models such as global water level simulations. The next decade should see significant advances in this field.
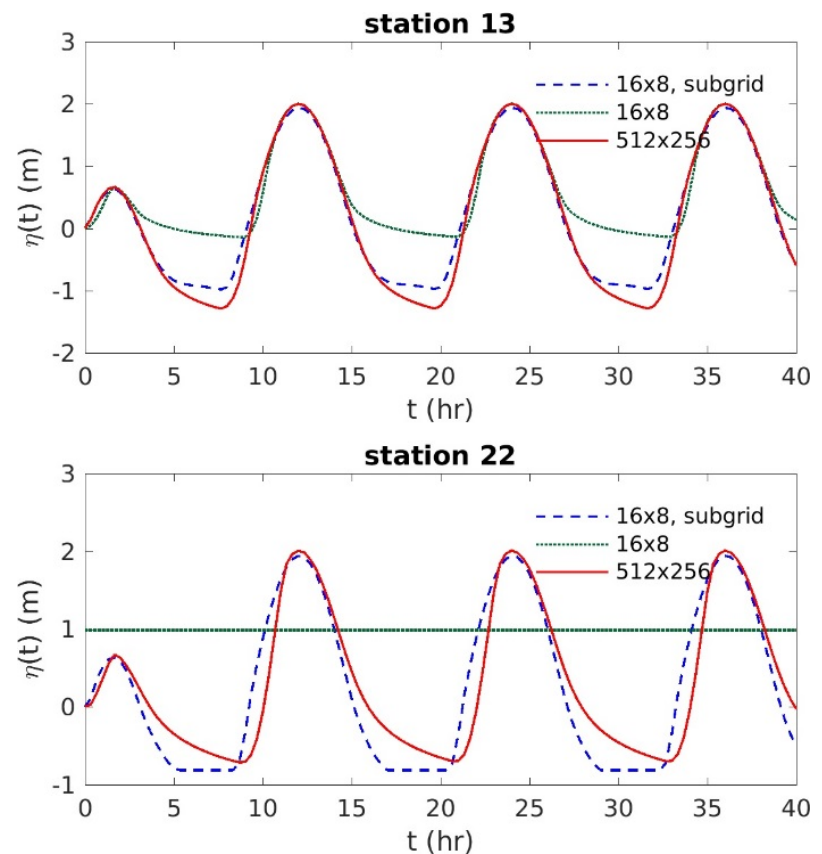

Figure 1. Tidal filling of Buttermilk Bay, MA. Results for: High resolution simulation $(512 \times 256)$; Low resolution standard simulation $(16 \times 8)$; and Low resolution subgrid simulation (16×8, subgrid). Station 13 is in the main bay, and Station 22 is in a secondary bay with a very narrow connection to the main bay.

\section{REFERENCES}

Casulli (2009). A high-resolution wetting and drying algorithm for free-surface hydrodynamics, Int. J. Num. Meth. Fluids, WILEY, vol. 60, pp. 32-39. 